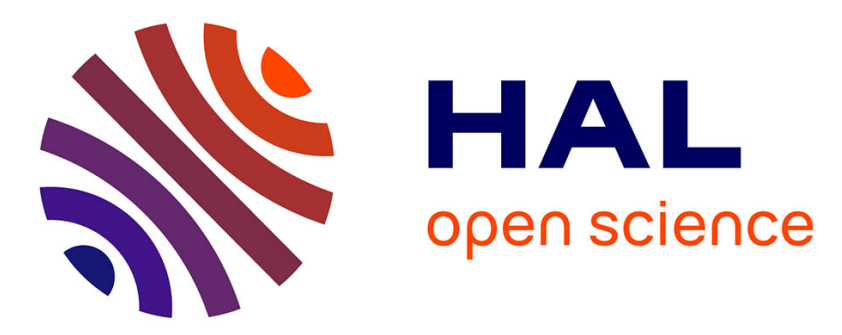

\title{
Comparing Weak and Strong PEEC-MoM Coupling
}

Lounès Mokhtari, Benoît Delinchant, Thierry Chevalier, Jean-Louis Coulomb

\section{To cite this version:}

Lounès Mokhtari, Benoît Delinchant, Thierry Chevalier, Jean-Louis Coulomb. Comparing Weak and Strong PEEC-MoM Coupling. COMPUMAG 2009, Nov 2009, Florianopolis, Brazil. pp 83-84. hal00440316

\section{HAL Id: hal-00440316 https://hal.science/hal-00440316}

Submitted on 21 Jan 2019

HAL is a multi-disciplinary open access archive for the deposit and dissemination of scientific research documents, whether they are published or not. The documents may come from teaching and research institutions in France or abroad, or from public or private research centers.
L'archive ouverte pluridisciplinaire HAL, est destinée au dépôt et à la diffusion de documents scientifiques de niveau recherche, publiés ou non, émanant des établissements d'enseignement et de recherche français ou étrangers, des laboratoires publics ou privés. 


\title{
Comparing Weak and Strong PEEC-MoM Coupling
}

\author{
L. Mokhtari, B. Delinchant, T. Chevalier, and J-L. Coulomb \\ Grenoble Electrical Engineering Laboratory, \\ INPG/UJF CNRS UMR 5269, ENSE ${ }^{3}$, BP 46, 38402 Saint-Martin d'Hères, France \\ Lounes. MOKHTARI@G2ELab.grenoble-inp.fr
}

\begin{abstract}
The aim of this paper is to compare strong and weak coupling of the PEEC and MoM methods applied to the modeling of magnetoharmonic problem, and to generalize the weak coupling approach. MoM and PEEC are dedicated to the modeling of specific parts of the overall device. As an example of validation, we have chosen the modeling of a simple transformer. Resolutions of several weak couplings are compared to the strong coupling approach. A new strategy of weak coupling is proposed to improve the time of resolution.
\end{abstract}

Index Terms-PEEC method, MoM method, weak/strong coupling, iterative methods.

\section{INTRODUCTION}

$\mathbf{T}$ HE MODELING of multiphysic systems needs powerful modeling tools and simulation methods. In this context, a specialized electric and magnetic method will be coupled in order to model an electromagnetic device.

The PEEC (Partial Elements Equivalent Circuit) approach has been proved to be excellent for the modeling of many ranges of cabling and interconnections in electric structures. The ferromagnetic materials can be modeled by FEM or integral method like Method of Moments [1][2]. Couplings of these methods have been done successfully in [3][4][5].

In the following sections, different coupling strategies of PEEC and MoM method will be compared. The figure below (Fig.1) shows the strategies commonly used for coupling programs.

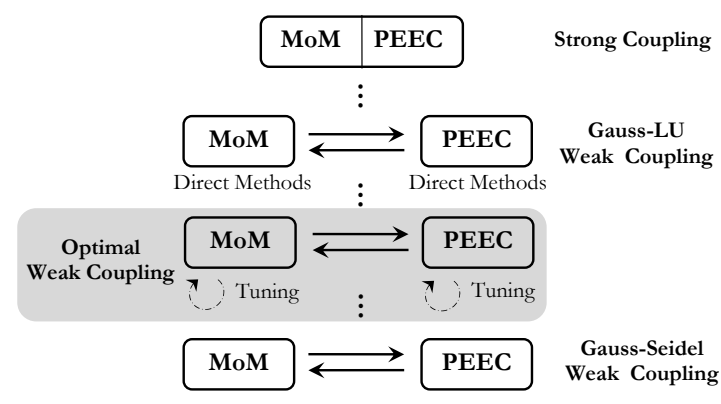

Fig. 1. Coupling strategies.

We have proposed an Optimal Weak Coupling strategy based on tuning models to improve the simulation performances. Both iterative and direct methods will be used to solve each of strong and weak coupling.

\section{PEEC AND MOM METHOD}

\section{A. The PEEC method}

The principle of the PEEC method is to decompose electric circuits into several parts, then calculate the mutual between them also the contribution of each of these elementary circuits to the total inductance [5]. The partial mutual between two electric conductors $i$ and $j$ is calculated as follows (1)

$$
M p_{i j}=\frac{\mu_{0}}{4 . \pi} \cdot \frac{1}{S_{i} S_{j}} \int_{V_{j}} \int_{V_{i}} \frac{1}{r_{i}} \cdot d V_{i} \cdot d V_{j}
$$

From (1), we build the impedances matrix (2)

$$
Z_{m}=\left[\begin{array}{cc}
R_{1}+j \omega L_{1} & j \omega M_{12} \\
j \omega M_{21} & R_{2}+j \omega L_{2}
\end{array}\right]
$$

The impedances matrix can be obtained directly with using software as INCA3D to the modeling of the conductors and a specific solver to extract the impedance matrix [6].

The source voltage formulation is defined as follows (3)

$$
U=Z_{m} \cdot I+f e m
$$

where fem is the well known electromotive force induced by the electric current $I$ in the conductors.

\section{B. The Method of Moments}

The MoM is an integral approach well adapted to the modeling of magnetic and radiating regions with simple geometries [1]. The global elements magnetic field is given by (4)

$$
H=H_{\text {ext }}+H_{\text {red }}
$$

where $H_{e x t}$ and $H_{\text {red }}$ are respectively the external magnetic field generated by the source field and the reaction of the material to the source field. In case of linear and unsaturable material, the discretization gives us the magnetic field in any point $P$ of the material (5).

$$
H(P)=H_{e x t}(P)+\frac{1}{4 \pi} \iiint_{V_{\text {mag }}}\left(3 \frac{\vec{M} \cdot \vec{r}}{r^{5}} \vec{r}-\frac{\vec{M}}{r^{3}}\right) \cdot d V_{\text {mag }}
$$

With making $M$ (Magnetization of the element $P$ ) out of the second term of the equation (5), we write the formulation of the magnetic field for all mesh elements (6)

$$
[H]=\left[H_{\text {ext }}\right]+[g] \cdot[M]
$$


The matrix $[g]$ is called the magnetostatic matrix, it represents the interactions between the material elements. It can be obtained directly with using some softwares specialized in the modeling of magnetic devices like LOCAPI [6].

\section{Coupling terms}

\section{Lenz-Faradays law}

The impact of the material magnetization $[M]$ on the conductor is the induced electromotive force $[\mathrm{fem}]$. The vector potential created by $[M]$ is given by (7)

$$
\vec{A}=\frac{\mu_{0}}{4 \pi} \iiint_{V_{\text {mag }}} \frac{\vec{M} \times \vec{r}}{\|r\|^{3}} \cdot d V_{\text {mag }}
$$

The link between the potential vector and the electromotive force is given below (8)

$$
f e m=j \omega \int_{l_{c}} \vec{A} \cdot d l_{c}
$$

The matrix formulation of (8) is

$$
[\mathrm{fem}]=[L F] \cdot[M]
$$

where $[L F]$ is calculated with the geometric parameters of the system $r$ and $V_{\text {mag }}$.

\section{Biot-Savarts law}

The impact of the conductor current $I$ on the material is the excitation magnetic field $H_{\text {ext }}$. The magnetic field created by the electric current $I$ is given by (9)

$$
H_{\text {ext }}(P)=\frac{1}{4 \pi} \iiint_{V_{\text {cond }}} \frac{\overrightarrow{J_{s}} \times \vec{r}}{\|r\|^{3}} \cdot d V_{\text {cond }}
$$

where $\vec{J}_{s}$ is the uniform density of the electric current $I$ in the conductor. The expression of $H_{e x t}$ in (10) becomes

$$
\left[H_{\text {ext }}\right]=[B S] \cdot[I]
$$

where $[B S]$ is calculated with the geometric parameters of the system.

\section{THE PEEC-MOM COUPLING}

The modeling of electromagnetic devices requires the modeling of the electrical and magnetic part, but also the interactions between them. This interaction is usually called "Coupling".

\section{A. Weak Coupling}

In this coupling, each method is available separately (Fig.2). The electric and magnetic systems are solved successively, the resolution of one is injected into the other and so that until achievement of the accuracy requirements.

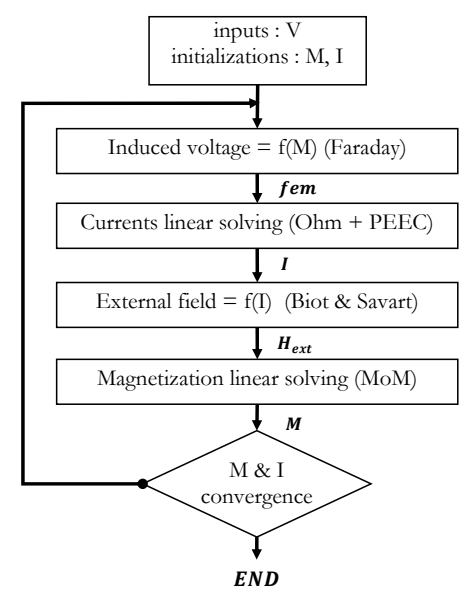

Fig. 2. Algorithm of weak coupling iterative approach.

\section{B. Strong Coupling}

The strong PEEC-MoM coupling developed in [2] aimed at grouping in one global matrix the overall modeling as detailled below.

We replace $H_{\text {ext }}$ in (6) by its formulation in (11), we obtain

$$
[H]=[B S][I]+[g][M]
$$

The material law says that $\left(\mu_{r}-1\right) H=M$ so if we multiply the equation (12) by $\left(\mu_{r}-1\right)$, we obtain

$$
\left(\left[I_{d}\right]-\left(\mu_{r}-1\right)[g]\right)[M]-\left(\mu_{r}-1\right)[B S][I]=0
$$

Lets consider : $\left\{\begin{array}{l}\left(\left[I_{d}\right]-\left(\mu_{r}-1\right)[g]\right)=[M M] \\ -\left(\mu_{r}-1\right)[B S]=\left[B S_{m}\right]\end{array}\right.$

then (13) becomes

$$
[M M][M]+\left[B S_{m}\right][I]=0
$$

The electric model (15) is built by replacing the formulation of $f e m$ in (3) by that of (9)

$$
[L F][M]+\left[Z_{m}\right][I]=U
$$

Now, it is possible to group the magnetic (14) and electric (15) model in one global matrix system, which represents the overall model of the system (16)

$$
\left[\begin{array}{cc}
M M & B S_{m} \\
L F & Z_{m}
\end{array}\right] \cdot\left[\begin{array}{c}
M \\
I
\end{array}\right]=\left[\begin{array}{c}
0 \\
U
\end{array}\right]
$$

The resolution of the system (16) gives as $\left[\begin{array}{l}M \\ I\end{array}\right]$ vector of unknowns composed by magnetizations of the material elements and the electric currents in the conductors, according to the sources vector $\left[\begin{array}{c}0 \\ U\end{array}\right]$. 


\section{APPLICATION}

\section{A. Device description}

The application example is the modeling of a simple transformer (Fig.2) with 2 current unknowns and a variable number of magnetization unknowns from 500 to 2000. Two square conductors are placed on two parallel planes and a magnetic core is placed through their center. The first conductor is powered by an alternative source voltage $(1 \mathrm{~V}, 1 \mathrm{kHz})$. The second conductor is short-circuited.

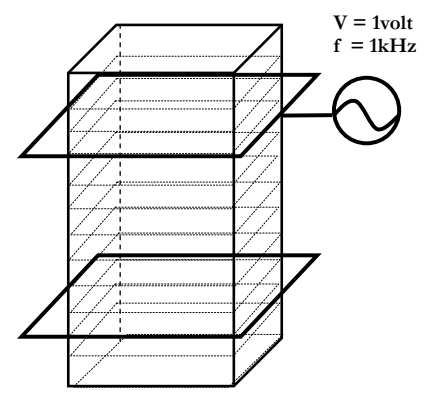

Fig. 3. Geometry of the test device.

Note that the coupling models have been validated by a finit element approach. For the same results of currents and magnetizations, the time resolution and convergence between the strong and weak coupling are compared.

\section{B. First Results}

The curves of the first results are plotted in the figure below (Fig.4).

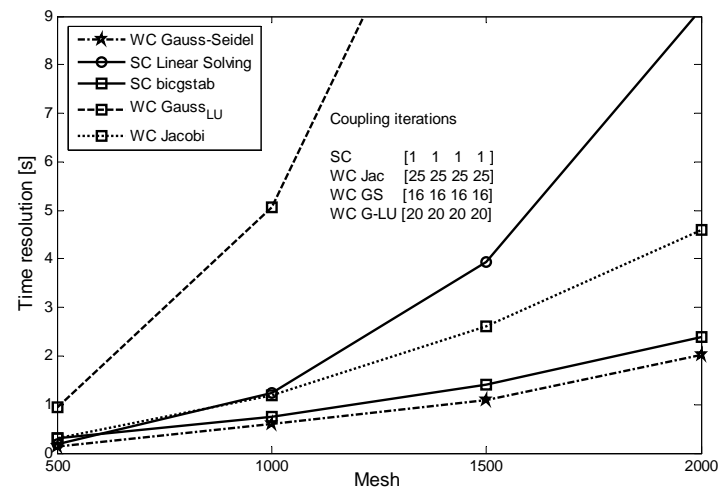

Fig. 4. Performances of the methods.

\section{Conclusion1}

For a little mesh elements, the strong coupling using direct methods is a good solution but for a fine mesh, the iterative method like BiConjugate Gradient Stabilized Method is better adapted.

The Gauss Seidel method combined with the weak coupling shows very good performances comparing to the bicgstab method because the subsystems conditioning in the weak coupling is better than the conditioning of the overall system in the strong coupling.

\section{Convergence Study}

Here, the convergence and the stability of the different methods are studied and discussed.
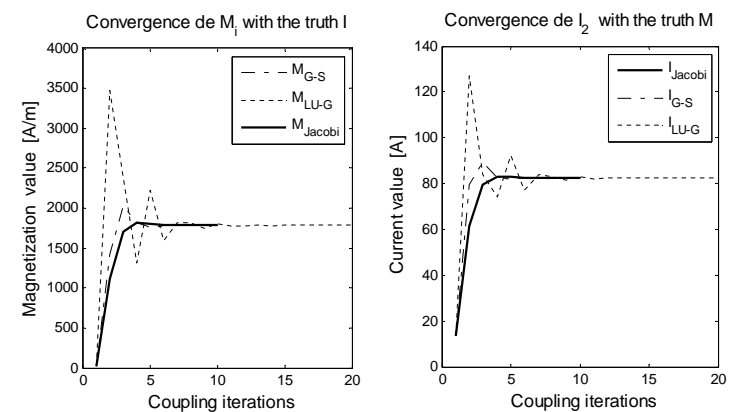

Fig. 5. Convergence of LU-Gauss, Jacobi and Gauss-Seidel method.

About the stability of the methods, oscillations appear when using the LU-Gauss method (Fig.4) and the convergence of the model is delayed until the $10^{t h}$ coupling iteration, unlike the Jacobi and Gauss-Seidel method. The oscillations persist even with using initial values very close to the truth values of magnetization and electric currents.

\section{Conclusion 2}

The weak coupling using iterative methods like Jacobi and Gauss-Seidel are more stable and converge faster than WC LU-Gauss method. The convergence of the WC LU-Gauss can be improved with using a parametric relaxation on the magnetization and current.

\section{Optimal parameters}

In the weak coupling built before (Fig.1), for each coupling iteration, we compute only once the electric and magnetic model. We add two tuned loops to solve more than once the electric and magnetic model (Fig.6). Now, for one coupling iteration, we make iter $M$ and iter $I$ iterations on respectively the magnetic and electric model.

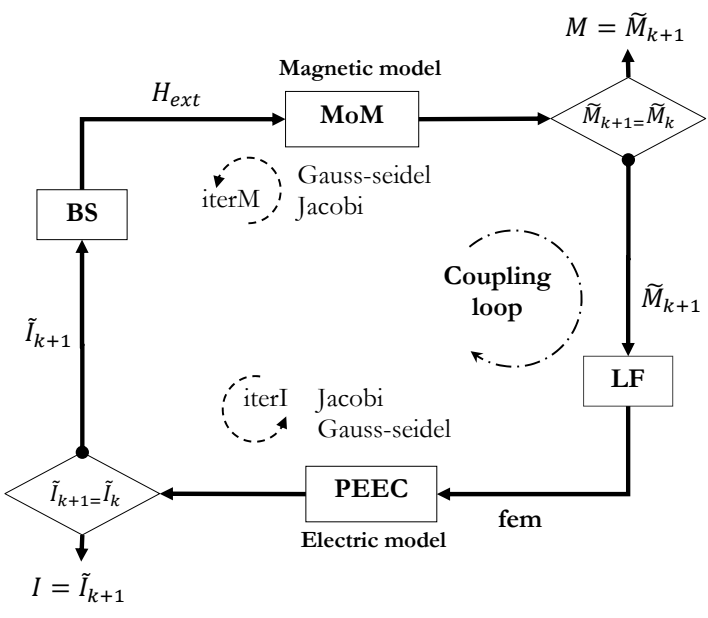

Fig. 6. Adjustabe Weak coupling loop.

A test for 2000 mesh elements was performed to identify if there is an optimal number of iteration can be made locally on 
the electric and magnetic model to reduce the time resolution.

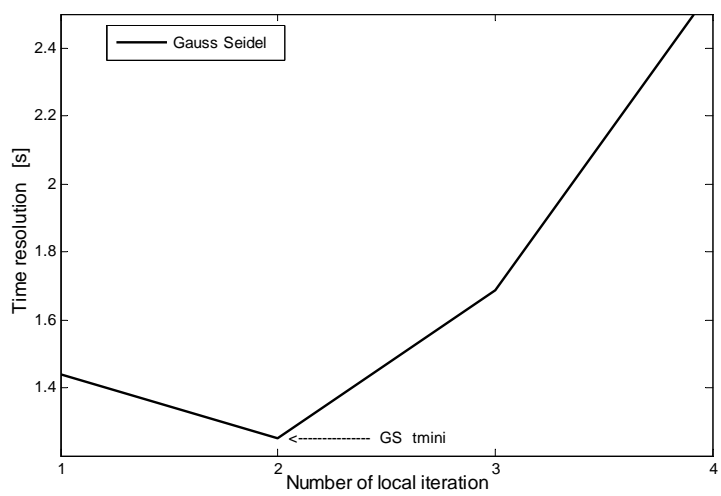

Fig. 7. Time resolution of WC G-S regarding the local iteration number.

From (Fig.7), the optimal number of iteration that reduces the most the time resolution is 2 for Gauss-Seidel method. An algorithm was implemented to find these parameters so the overall model is automatically tuned with the optimal parameters.

\section{E. Improvement of time resolution}

The overall model of the system is tuned with the optimal parameters and solved. In the figure below (Fig.8), we have plotted the new curves of time resolution regarding the number of mesh elements and given the number of coupling iterations. In the same figure we have let appear the curves obtained before in order to compare between them.

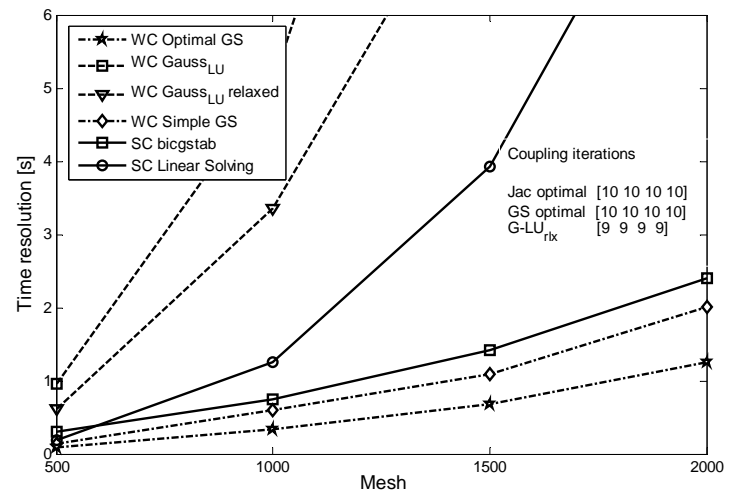

Fig. 8. Performances of the methods tuned with optimal parameters.

\section{Conclusion 3}

By using the optimal parameters, we have reduced the time resolution of the model comparing to the previous weak coupling.

We have also minimized the number of iterations on the coupling loop which is very important when coupling softwares on network and where an iteration on coupling takes a long time.

\section{CONCLUSION}

Different strategies of weak coupling of the PEEC and MoM methods have been compared with the strong coupling. A new strategy of weak coupling (Fig.6) is proposed, the results obtained prove that our weak coupling is:

1) better in term of time resolution than the strong one. We also improved the gain of the first weak coupling (Fig.2) by $27 \%$ and $17 \%$ for respectively Gauss-seidel and jacobi methods;

2) more stable than the weak coupling using direct methods; it speeds and relaxes the convergence of the model and

3) minimizes the iteration on coupling loop, which is very important in practice where we couple programs on network.

Adding to these gains, this strategy presents other advantages like, easy understanding because it repeats faithfully the evolution of physical phenomena in the system, more flexible, parallelizable tasks, and gives us the possibility to reuse the codes developed.

\section{REFERENCES}

[1] O. Chadebec, J-L. Coulomb, and F. Janet, A Review of Magnetostatic Moment Method, IEEE Transactions on Magnetics, Vol. 42, no. 4, april 2006.

[2] G. Antonini, M. Sabatini, G. Miscione, PEEC modeling of linear magnetic materials. IEEE International Symposium on Electromagnetic Compatibility, EMC 2006. Vol. 1, n14-18 Aug. 2006, pp: 93 - 98

[3] T. Le Duc, O. Chadebec, J-M. Guichon and G. Meunier, Couplage de la méthode moment magnétique avec la mt́hode PEEC pour la rsolution des problmes magnètodynamique, NUMELEC 2008, Lige (Belgique) 8-9-10 dcembre 2008.

[4] M. Troscher, U. Jakobus, G. Steinmair, Combined PEEC/MoM solution technique for efficient electromagnetic emission calculations of PCBs in metallic enclusors, International Symposium on Electromagnetic Compatibility, EMC 2004.

[5] T-S. Tran, G. Meunier, P. Labie, Y. Le Floch, J. M. Guichon, J. Roudet, FEM-PEEC coupled method for modeling solid conductors in the presence of ferromagnetic material, COMPEL 2008, EMC 2006. Vol. 27, Issue 4, pp $904-910$.

[6] http://www.g2elab.grenoble-inp.fr 